\title{
Zeros and orthogonality of the Askey-Wilson polynomials for $q$ a root of unity ${ }^{1}$
}

\author{
Vyacheslav Spiridonov and Alexei Zhedanov
}

\begin{abstract}
We study some properties of the Askey-Wilson polynomials (AWP) when $q$ is a primitive $N$ th root of unity. For general four-parameter AWP, zeros of the $N$ th polynomial and the orthogonality measure are found explicitly. Special subclasses of the AWP, e.g., the continuous $q$-Jacobi and big $q$-Jacobi polynomials, are considered in detail. A set of discrete weight functions positive on a real interval is described. Some new trigonometric identities related to the AWP are obtained. Normalization conditions of some polynomials are expressed in terms of the Gauss sums.
\end{abstract}

Key words. q-orthogonal polynomials, roots of unity, Gauss sums

AMS Subject Classifications. 33D45 (Primary) 11L05 (Secondary)

\section{Introduction}

The theory of orthogonal polynomials of one variable is based upon the three-term recurrence relation $[8]$

$$
P_{n+1}(x)+b_{n} P_{n}(x)+u_{n} P_{n-1}(x)=x P_{n}(x), \quad n=1,2, \ldots
$$

with the initial conditions

$$
P_{0}(x)=1, \quad P_{1}(x)=x-b_{0} .
$$

There is a representation theorem concerning orthogonality of the polynomials $P_{n}(x)$ with respect to some complex measure $\mu(x)$ in case $u_{n}$ and $b_{n}$ are arbitrary complex coefficients; see, e.g., $[3,8]$,

$$
\int_{C} P_{n}(x) P_{m}(x) d \mu(x)=h_{n} \delta_{n m},
$$

where $h_{n}=u_{1} u_{2} \cdots u_{n}$ and $C$ is some contour in the complex plane. When $b_{n}$ are real and $u_{n}>0$, the measure can be chosen real-valued (then $C$ is the real line) and nondecreasing. For such cases it can be shown that all zeros $x_{s}, s=0,1, \ldots, N-1$, of any polynomial $P_{N}(x)$ are real and simple [8]. Moreover, these zeros can be used for construction of a discrete orthogonality relation for polynomials of the degree lower than $N$ :

$$
\sum_{s=0}^{N-1} P_{n}\left(x_{s}\right) P_{m}\left(x_{s}\right) w_{s}=h_{n} \delta_{n m},
$$

where $n, m=0,1, \ldots, N-1$ and the weight function is

$$
w_{s}=\frac{h_{N-1}}{P_{N-1}\left(x_{s}\right) P_{N}^{\prime}\left(x_{s}\right)} .
$$

${ }^{1}$ Date: April 1996; published in Duke Math. J. 89 (1997), 283-305. 
The property (1.4) was proven in [4] under the assumption that $b_{n}$ are real and $u_{n}$ are positive. However, the proof remains valid even in general cases of complex $b_{n}, u_{n}$. The only requirement to be added is that all zeros $x_{s}$ must be simple which is not guaranteed if the recurrence coefficients are complex. For example, for $b_{n}=0$ one finds from the recurrence relation $(1.1), P_{3}(x)=x\left(x^{2}-u_{1}-u_{2}\right)$. If $u_{2}=-u_{1}$ then all zeros of the polynomial $P_{3}(x)$ are located at $x=0$. It is thus always necessary to check simplicity of the zeros $x_{s}$ in order for (1.4) to be applicable.

In this paper we present a study of the finite orthogonality property (1.4) of the Askey-Wilson polynomials (AWP) for the case when $q$ is a root of unity. Recall that the recurrence coefficients for AWP have the form $[3,9]$

$$
u_{n}=\xi_{n} \eta_{n-1}, \quad b_{n}=-\xi_{n}-\eta_{n}+a+a^{-1}
$$

where

$$
\begin{aligned}
& \xi_{n}=\frac{a\left(1-q^{n}\right)\left(1-b c q^{n-1}\right)\left(1-b d q^{n-1}\right)\left(1-c d q^{n-1}\right)}{\left(1-g q^{2 n-2}\right)\left(1-g q^{2 n-1}\right)} \\
& \eta_{n}=\frac{a^{-1}\left(1-a b q^{n}\right)\left(1-a c q^{n}\right)\left(1-a d q^{n}\right)\left(1-g q^{n-1}\right)}{\left(1-g q^{2 n}\right)\left(1-g q^{2 n-1}\right)}
\end{aligned}
$$

and $g=a b c d$. The parameters $a, b, c, d$ are arbitrary complex numbers. The explicit expression of AWP in terms of the basic hypergeometric functions is $[3,9]$

$$
P_{n}(x)=D_{n 4} \varphi_{3}\left(\begin{array}{c}
q^{-n}, g q^{n-1}, a t, a / t \\
a b, a c, a d
\end{array} ; q, q\right)
$$

where

$$
D_{n}=\frac{a^{-n}(a b ; q)_{n}(a c ; q)_{n}(a d ; q)_{n}}{\left(g q^{n-1} ; q\right)_{n}}
$$

and $x=t+t^{-1}$. Here $(a ; q)_{n}$ is the $q$-shifted factorial defined as $(a ; q)_{0}=1$ and $(a ; q)_{n}=(1-a)(1-$ $a q) \cdots\left(1-a q^{n-1}\right)$ for integer $n>0$. The general $q$-hypergeometric series is defined as follows [9]:

$$
{ }_{r} \varphi_{s}\left(\begin{array}{c}
a_{1}, a_{2}, \ldots, a_{r} \\
b_{1}, \ldots, b_{s}
\end{array} ; q, z\right)=\sum_{n=0}^{\infty} \frac{\left(a_{1}, a_{2}, \ldots, a_{r} ; q\right)_{n}}{\left(q, b_{1}, \ldots, b_{s} ; q\right)_{n}}\left[(-1)^{n} q^{n(n-1) / 2}\right]^{1+s-r} z^{n}
$$

where the compact notation $\left(a_{1}, a_{2}, \ldots, a_{r} ; q\right)_{n}=\left(a_{1} ; q\right)_{n} \cdots\left(a_{r} ; q\right)_{n}$ is used.

For real $q$ and appropriate restrictions upon the parameters $a, b, c, d$ the AWP are orthogonal on a finite interval of the real axis with a positive continuous weight function [3]. In this case, AWP satisfy a difference equation defined upon the hyperbolic grid [3, 15]. A possibility of constructing orthogonal polynomials for trigonometric grids has been mentioned briefly in [15]. In this paper we concentrate on the case when $q$ is a primitive $N$-th root of unity:

$$
q=\exp (2 \pi i M / N)
$$

where $M$ and $N$ are mutually co-prime positive integers, $M<N$. We consider only finite sets of orthogonal polynomials with discrete measures which are different from the so-called sieved polynomials (see, e.g., [1]).

\section{Zeros of the Nth Askey-Wilson polynomial}

In this section we analyze location of zeros for generic $N$ th order AWP depending on four parameters for $q$ a primitive $N$ th root of unity. 
Consider the explicit form of $P_{N}(x)(1.9)$ for $q$ fixed by (1.12). Obviously we cannot use this expression directly, because the factor $\left(q^{-N} ; q\right)_{N} /(q ; q)_{N}$ in the $N$ th term of the series is indeterminate. Nevertheless, we can apply a limiting procedure taking $q=\exp (\epsilon+2 \pi i M / N)$ and going to the limit $\epsilon \rightarrow 0$. Then it is easy to see that

$$
\lim _{\epsilon \rightarrow 0} \frac{\left(q^{-N} ; q\right)_{N}}{(q ; q)_{N}}=\lim _{\epsilon \rightarrow 0}(-1)^{N} q^{-N(N+1) / 2}=-1 .
$$

Using (2.1) one can find that

$$
P_{N}(x)=D_{N}\left(1-\frac{(g ; q)_{N}(a t ; q)_{N}\left(a t^{-1} ; q\right)_{N}}{(a b ; q)_{N}(a c ; q)_{N}(a d ; q)_{N}}\right)
$$

Since $u_{N}=0$ in (1.1). we have a finite set of polynomials and it is not necessary to consider ambiguities in the higher order terms in the series (1.9).

The $q$-shifted factorial in (2.2) can be simplified because for any complex parameter $a$ one has the identity (which is valid only for $q$ a root of unity)

$$
(a ; q)_{N}=1-a^{N} .
$$

Indeed, $(a ; q)_{N}=(1-a)(1-a q) \cdots\left(1-a q^{N-1}\right)$ is a $N$-th order polynomial in argument $a$. The zeros of this polynomial are $1, q, q^{2}, \ldots, q^{N-1}$, whence (2.3) follows.

We can rewrite $(2.2)$ in the form

$$
P_{N}(x)=t^{N}+t^{-N}-E_{N}
$$

where $E_{N}$ is a rational function of parameters,

$$
E_{N}=\frac{a^{N}+b^{N}+c^{N}+d^{N}-(a b c)^{N}-(b c d)^{N}-(a b d)^{N}-(a c d)^{N}}{1-(a b c d)^{N}}
$$

We call $E_{N}$ a combinatorial invariant because its symmetry with respect to permutation of the parameters $a, b, c, d$ plays an important role in the following.

From (2.4) it is not difficult to find zeros of $P_{N}(x)$ :

$$
x_{s}=r q^{s}+r^{-1} q^{-s}, \quad s=0,1, \ldots, N-1,
$$

where for simplicity we assume that $r$ is the root with minimal argument of the equation

$$
r^{N}=E_{N} / 2+\sqrt{E_{N}^{2} / 4-1}, \quad \text { or } \quad r^{-N}=E_{N} / 2-\sqrt{E_{N}^{2} / 4-1} .
$$

Let us point out a curious identity associated with the combinatorial invariant $E_{N}$ :

$$
\frac{\left(1-a^{N} r^{N}\right)\left(1-b^{N} r^{N}\right)\left(1-c^{N} r^{N}\right)\left(1-d^{N} r^{N}\right)}{\left(a^{N}-r^{N}\right)\left(b^{N}-r^{N}\right)\left(c^{N}-r^{N}\right)\left(d^{N}-r^{N}\right)}=1 .
$$

Now we are ready to formulate the orthogonality relation for AWP. First we need the condition of simplicity of the zeros $x_{s}$, which is

$$
E_{N} \neq \pm 2 \text {. }
$$

Second, in order for the polynomials $P_{n}(x), 0 \leq n \leq N$, to be well defined we impose the constraints

$$
g, a b, a c, a d, b c, b d, c d \neq q^{k}, \quad k=0,1, \ldots, N-1,
$$


where $q$ is fixed by (1.12). These restrictions guarantee that $u_{n}$ does not vanish between two zeros at $n=0$ and $n=N$ or that $u_{n}, b_{n}$ do not blow up (cf. [16]). Under the conditions (2.9) one can calculate

$$
P_{N}^{\prime}\left(x_{s}\right)=N \frac{r^{N}-r^{-N}}{r q^{s}-r^{-1} q^{-s}}
$$

Using the same $\epsilon \rightarrow 0$ limiting procedure as in (2.1) for (unambiguous) determination of the polynomial $P_{N-1}\left(x_{s}\right)$ we get the following

Theorem 1. The weight function of the finite-dimensional set of Askey-Wilson polynomials for $q$ a primitive root of unity (1.12) and the constraints upon the parameters (2.8), (2.9) has the form

$$
w_{s}=\frac{h_{N-1}\left(r q^{s}-r^{-1} q^{-s}\right)}{N D_{N-1}\left(r^{N}-r^{-N}\right) F(s)},
$$

where $s=0,1, \ldots, N-1$, and

$$
F(s)=\sum_{n=0}^{N-1} \frac{\left(g q^{-2}, a r q^{s}, a r^{-1} q^{-s} ; q\right)_{n}}{(a b, a c, a d ; q)_{n}} q^{n}
$$

This form of the weight function is not convenient for the analysis of its positivity since a truncated ${ }_{4} \varphi_{3}$ series (2.12) depending on the discrete argument $s$ is involved into the definition. In the next section we shall derive a more explicit form of $w_{s}$.

\section{Difference equation and absence of self-duality}

In this section we derive a difference equation for generic AWP for $q$ a root of unity and show that a complete self-duality property (a permutational symmetry between the parametrization of polynomial's argument and its degree) is absent.

In order to derive this difference equation we use contiguous relations for the terminating basic hypergeometric series ${ }_{4} \varphi_{3}$. Recall that if one denotes

$$
\phi(\xi, \eta)={ }_{4} \varphi_{3}\left(\begin{array}{c}
\xi, \eta, \gamma, \delta \\
e, f, h
\end{array} ; q, q\right)
$$

where one of the parameters $\xi, \eta, \gamma, \delta$ is equal to $q^{-M}, M$ a positive integer, and $e f h=\xi \eta \gamma \delta q$, then one has the following relation $[2,9]$ :

$$
B_{1} \phi\left(\xi q^{-1}, \eta q\right)+B_{3} \phi(\xi, \eta)+B_{2} \phi\left(\xi q, \eta q^{-1}\right)=0
$$

where

$$
\begin{aligned}
& B_{1}=\eta(1-\eta)(\xi q-\eta)(\xi-e)(\xi-f)(\xi-h), \\
& B_{2}=\xi(1-\xi)(\xi-\eta q)(\eta-e)(\eta-f)(\eta-h), \\
& B_{3}=\xi \eta(\xi-\eta q)(\xi q-\eta)(\xi-\eta)(1-\gamma)(1-\delta)-B_{1}-B_{2}
\end{aligned}
$$

In our case one should choose the parametrization

$$
\xi=a r q^{s}, \eta=a r^{-1} q^{-s}, \gamma=q^{-n}, \delta=g q^{n-1}, e=a b, f=a c, h=a d,
$$


which gives $P_{n}\left(x_{s}\right)=D_{n} \phi\left(a r q^{s}, a r^{-1} q^{-s}\right)$. Substituting (3.5) into (3.1) we arrive at the difference equation for AWP with the argument $x_{s}$ given by (2.6):

$$
A_{s} P_{n}\left(x_{s-1}\right)+C_{s} P_{n}\left(x_{s+1}\right)-\left(A_{s}+C_{s}\right) P_{n}\left(x_{s}\right)=\lambda_{n} P_{n}\left(x_{s}\right)
$$

where

$$
\lambda_{n}=\left(q^{-n}-1\right)\left(1-g q^{n-1}\right)
$$

and

$$
\begin{aligned}
A_{s} & =\frac{g q^{-1}\left(1-r a^{-1} q^{s}\right)\left(1-r b^{-1} q^{s}\right)\left(1-r c^{-1} q^{s}\right)\left(1-r d^{-1} q^{s}\right)}{\left(1-r^{2} q^{2 s-1}\right)\left(1-r^{2} q^{2 s}\right)} \\
C_{s} & =\frac{\left(1-a r q^{s}\right)\left(1-b r q^{s}\right)\left(1-c r q^{s}\right)\left(1-d r q^{s}\right)}{\left(1-r^{2} q^{2 s}\right)\left(1-r^{2} q^{2 s+1}\right)}
\end{aligned}
$$

Let us note that after the (complex) shift of the variable $s$ such that $r a^{-1} q^{s} \rightarrow q^{s}$ and a change of notation for parameters, the equation (3.6) coincides with the three-term recurrence relation for AWP. This means that the difference equation (3.6) may be considered as a recurrence relation for a special class of associated AWP. The general associated AWP are determined by (1.1), (1.2) and the coefficients $u_{n}, b_{n}$ obtained from (1.6) after the replacement of $q^{n}$ by $\mu q^{n}$, where $\mu$ is an arbitrary complex parameter [11].

But the recurrence coefficients $A_{s}$ and $C_{s}$ do not have zeros for any $s=0,1, \ldots$, so that one can consider orthogonal polynomials of unlimited degree. The orthogonality measure of these polynomials will be continuous, i.e. the parameter $\lambda_{n}$ on the right hand side of (3.6) will not be quantized. Moreover, there exist regions of the parameters $a, b, c, d$ such that the coefficients $u_{n}$ are positive and $b_{n}$ are real. For instance, such a situation takes place if we set

$$
a=q^{\alpha}, \quad d=q^{\delta}, \quad b=-q^{\beta}, \quad c=-q^{\gamma}, \quad q=e^{2 \pi i / N},
$$

where $\alpha, \beta, \gamma, \delta$ are real nonzero parameters, choose $N$ even, and impose the restrictions

$$
|\alpha|,|\beta|,|\gamma|,|\delta| \ll 1, \quad \alpha<0, \quad \alpha<\beta<-\alpha, \quad \gamma>-\alpha, \quad \delta>-\alpha .
$$

Under these conditions the difference equation (3.6) becomes Hermitian. From this fact follows the existence of $q$-orthogonal polynomials (a special subclass of the associated AWP) for $q$ a root of unity with the continuous positive measure which are different from the sieved polynomials. Consideration of these polynomials lies beyond the scope of present work.

Before calculating the weight function, let us discuss a self-duality of the AWP [5, 14]. For simplicity let us limit consideration to finite sets of orthogonal polynomials with positive measures $P_{n}(s)$, where $n$ is the degree of polynomials and $s$ is the index numbering discrete values of the argument $x_{s}, n, s=0,1, \ldots, N-1>0$. Due to the Favard theorem, any set of such polynomials is uniquely determined by the three-term recurrence relation. As usual, one has some discrete orthogonality relations (1.4) and dual conditions:

$$
\sum_{n=0}^{N-1} \frac{P_{n}(s) P_{n}\left(s^{\prime}\right)}{h_{n}}=w_{s}^{-1} \delta_{s s^{\prime}}
$$

(see, e.g., [4]). In the dual picture, $P_{n}(s)$ are considered as eigenvectors with eigenvalues $\lambda_{n}$ of some Hermitian matrix acting upon the (dual) index $s$. The latter matrix is not necessarily of the Jacobi form; in general it is difficult to find explicitly. Moreover, it is not unique (there is a dependence on functional parametrization of $x_{s}$ on $s$ ). When this matrix is tridiagonal, one may refer to the 
corresponding polynomials as partially self-dual, because then both indices $n$ and $s$ can be considered as degrees of orthogonal polynomials. Polynomials $P_{n}(s)$ are called completely self-dual if there exists a symmetry such that $P_{n}(s)=P_{s}^{*}(n)$ (this relation requires a special normalization of polynomials, see $[5,14])$. Here the involution sign ${ }^{\prime * \prime}$ means a permutation of parameters of polynomials. In the simplest case there is no transformation of parameters at all and the symmetry condition is $P_{n}(s)=P_{s}(n)$, which means that $P_{n}(s)$, being considered as a $N \times N$ matrix, equals its transpose. For completely self-dual systems, the three-term recurrence relation in $n$ and the difference equation in $s$ coincide up to a change of parameters. In the $P_{n}(s)=P_{s}(n)$ case, these equations just totally coincide. For examples of such a situation we refer to the discrete series representations of the quantum algebra $s o_{q}(3)$ [17] describing $q$-ultraspherical polynomials at $q^{N}=1$.

In $[5,14]$ completely self-dual orthogonal polynomials were considered and it was proven that the only polynomials possessing this property are the $q$-Racah polynomials. The recurrence coefficients $\xi_{n}, \eta_{n}$ in (1.6) for these polynomials [2] satisfy the conditions $\xi_{0}=\eta_{N+1}=0$. For the case $q^{N}=1$ with generic values of parameters only the first factor $\xi_{n}$ creates zeros $\xi_{0}=\xi_{N}=0$. I.e., our $P_{n}\left(x_{s}\right)$ do not belong to the $q$-Racah polynomials and hence they are not completely self-dual. The latter can also be seen directly from the explicit expression (1.9): for $t=r q^{s}$ the permutation of $n$ and $s$ cannot be compensated by an appropriate normalization of polynomials and permutation of parameters. In fact, the values of variable $s$ can be extended from a finite set $s=0,1, \ldots, N-1$ to all integers without any problem. As a result, one gets the periodicity property $P_{n}\left(x_{s+N}\right)=P_{n}\left(x_{s}\right)$ and the same periodicity is satisfied by the coefficients $A_{s}, C_{s}$ in the difference equation (3.6). Evidently, these $A_{s}, C_{s}$ never vanish. $A_{s} C_{s-1} \neq 0$, so that there is not automatic reduction of the difference equation to the finite-dimensional matrix form in contrast to the $q$-Racah polynomials case.

As seen from (1.9), the polynomials $P_{n}\left(x_{s}\right)$ become completely self-dual if the condition $r=a q^{j}$, $j$ integer, is fulfilled. But this equality holds only if one of the products $a b, a c, a d$ is equal to $q^{k}, k$ integer, as it follows from (2.5). However, such constraints correspond to the $q$-Racah polynomials and they are forbidden in our case (2.9). To summarize the above considerations: duals of the AWP for $q^{N}=1$ are given by the special types of the associated AWP for $q^{N}=1$, i.e., there is no complete self-duality.

Let us return now to the problem of calculation of the weight function $w_{s}$. Existence of the difference equation (3.6) allows us to find a simpler form of $w_{s}$ than (2.11). Indeed, multiplying equation (3.6) by a function $\sigma(s)$ satisfying the only requirement of periodicity $\sigma(s+N)=\sigma(s)$, and combining it with a similar equation for the polynomials $P_{m}\left(x_{s}\right)$, one can get a bilinear relation:

$$
\begin{array}{r}
A_{s} \sigma(s)\left[P_{n}\left(x_{s-1}\right) P_{m}\left(x_{s}\right)-P_{n}\left(x_{s}\right) P_{m}\left(x_{s-1}\right)\right] \\
+C_{s} \sigma(s)\left[P_{n}\left(x_{s+1}\right) P_{m}\left(x_{s}\right)-P_{n}\left(x_{s}\right) P_{m}\left(x_{s+1}\right)\right] \\
=\left(\lambda_{n}-\lambda_{m}\right) \sigma(s) P_{n}\left(x_{s}\right) P_{m}\left(x_{s}\right) .
\end{array}
$$

Choose $\sigma(s)$ in such a way that

$$
A_{s+1} \sigma(s+1)=C_{s} \sigma(s)
$$

Then the equation (3.13) can be rewritten in the form

$$
R_{n m}(s+1)-R_{n m}(s)=\left(\lambda_{n}-\lambda_{m}\right) \sigma(s) P_{n}\left(x_{s}\right) P_{m}\left(x_{s}\right)
$$

where

$$
R_{n m}(s)=A_{s} \sigma(s)\left[P_{n}\left(x_{s}\right) P_{m}\left(x_{s-1}\right)-P_{n}\left(x_{s-1}\right) P_{m}\left(x_{s}\right)\right] .
$$

Summing (3.15) from $s=0$ to $s=N-1$ and using the obvious periodicity property $R_{n m}(s+N)=$ $R_{n m}(s)$, one gets the orthogonality relation:

$$
\left(\lambda_{n}-\lambda_{m}\right) \sum_{s=0}^{N-1} \sigma(s) P_{n}\left(x_{s}\right) P_{m}\left(x_{s}\right)=0, \quad n \neq m .
$$


The weight function $\sigma(s)$ is determined from the relation (3.14) uniquely, up to a multiplicative factor. Hence one should have $w_{s}=\kappa \sigma(s)$ where $\kappa$ is some constant and $w(s)$ is the weight function calculated in (2.11). From (3.14) we find:

$$
w_{s}=w_{0}(q / g)^{s} \frac{\left(1-r^{2} q^{2 s}\right)(a r, b r, c r, d r ; q)_{s}}{\left(1-r^{2}\right)(q r / a, q r / b, q r / c, q r / d ; q)_{s}},
$$

where $w_{0}$ is a normalization constant. Note that the consistency condition $\sigma(N)=\sigma(0)$ imposed upon $q$-difference equation (3.14) for $q^{N}=1$ (see [6] for a general discussion of such conditions) is satisfied in a quite nontrivial manner due to (2.7).

We have found thus the explicit dependence of the weight function $w_{s}$ on the discrete argument $s$. The remaining value $w_{0}$ can be evaluated from $(2.11)$

$$
w_{0}=a^{N-1} \frac{\left(1-(b c)^{N}\right)\left(1-(c d)^{N}\right)\left(1-(b d)^{N}\right)(1-g / q)\left(1-g / q^{2}\right)\left(r-r^{-1}\right)}{F(0)\left(1-g^{N}\right)^{2}(1-b c / q)(1-c d / q)(1-b d / q)\left(r^{N}-r^{-N}\right)},
$$

where $F(0)$ is fixed by $(2.12)$ at $s=0$. The normalization condition

$$
\sum_{s=0}^{N-1} w_{s}=1
$$

where $w_{s}$ is given by (3.18), leads to some non-trivial trigonometric identities which we summarize into the following theorem.

Theorem 2. Under the constraints (2.8), (2.9) upon the complex parameters $a, b, c, d$ and for $q=e^{2 \pi i M / N},(N, M)=1$, the following trigonometric identity takes place

$$
\begin{gathered}
\sum_{s=0}^{N-1}\left(\frac{q}{g}\right)^{s} \frac{\left(1-r^{2} q^{2 s}\right)(a r, b r, c r, d r ; q)_{s}}{(q r / a, q r / b, q r / c, q r / d ; q)_{s}} \\
=\frac{r^{1-N}\left(1-r^{2 N}\right)\left(1-g^{N}\right)^{2} f(a, b, c, d)}{(a b, a c, a d, b c, c d, b d ; q)_{N-1}(1-g / q)\left(1-g / q^{2}\right)},
\end{gathered}
$$

where

$$
f(a, b, c, d) \equiv \frac{(a b, a c, a d ; q)_{N-1}}{a^{N-1}} \sum_{n=0}^{N-1} \frac{\left(g q^{-2}, a r, a r^{-1} ; q\right)_{n}}{(a b, a c, a d ; q)_{n}} q^{n}
$$

Comments. Probably the truncated ${ }_{4} \varphi_{3}$ series (3.22) has a simpler representation through some finite products due to the specific properties of the roots of unity and algebraic relation between $r$ and $a, b, c, d$, but we were not able to find it. Since the AWP are symmetric with respect to permutation of parameters, the same symmetry should be valid for $f(a, b, c, d)$, but it has not been proved yet to be explicit. Since the left hand side of (3.21) is finite for $a \rightarrow 0$, the function (3.22) should be finite in this limit as well. The above identity looks as a root-of-unity analog of a special case of the Watson transformation formula for ${ }_{8} \varphi_{7}$ series [9], but the complete coincidence is not yet established.

The relation (3.21) exists only for $q$ a root of unity, i.e., there are no its analogs for other values of $q$ contrary to the $q$-Racah polynomials' weight function normalization condition holding for $|q|<1,|q|>1$, or $|q|=1$. As shown below (see also the Appendix), at the bottom of trigonometric identities obtained for $q^{N}=1$ like (3.21), one finds Gauss sums or similar summation formulas. The latter play an important role in the number theory. Therefore, the arithmetic aspects of (3.21) require a detailed investigation. Note in passing that the most general identity of such type should be tied to the biorthogonal rational functions associated with the special ${ }_{10} \varphi_{9}$ series [10]. 


\section{Two parameter symmetric Askey-Wilson polynomials}

In this section we consider a special subclass of the symmetric AWP, and determine some regions of parameters for which the measure is positive.

Consider AWP with $a=-c=q^{\alpha}, d=-b=q^{\beta}$. Then in the recurrence relation for AWP only nondiagonal terms survive, i.e., the polynomials are symmetric:

$$
P_{n+1}(x)+u_{n} P_{n-1}(x)=x P_{n}(x),
$$

where $n=0,1, \ldots, N-1$,

$$
u_{n}=\frac{4 \sin \omega n \sin \omega(n+2 \alpha+2 \beta-2) \cos \omega(n+2 \alpha-1) \cos \omega(n+2 \beta-1)}{\sin 2 \omega(n+\alpha+\beta-3 / 2) \sin 2 \omega(n+\alpha+\beta-1 / 2)}
$$

and $\omega=\pi M / N$.

One can show that if $M=1$ and $N$ is odd, the recurrence coefficients $u_{n}$ are positive, $u_{n}>0$, for $n=1,2, \ldots, N-1$, under the following constraints upon the parameters $\alpha$ and $\beta$ :

$$
-1 / 4<\alpha<1 / 4, \quad 1 / 4<\beta<3 / 4,
$$

or

$$
1 / 4<\alpha<3 / 4, \quad 3 / 4<\beta<5 / 4
$$

These restrictions, or their partners obtained after permuting the parameters $\alpha$ and $\beta$, provide only particular examples of positive $u_{n}$, the problem of finding all such cases (including the cases of $M \neq 1$ and even $N$ ) is complicated and is not considered here.

The difference equation for the taken symmetric AWP has the form (3.6), where

$$
\begin{gathered}
A_{s}=\frac{\sin 2 \omega(s-\alpha+1 / 4) \sin 2 \omega(s-\beta+1 / 4)}{\sin 2 \omega(s+1 / 4) \sin 2 \omega(s-1 / 4)}, \\
C_{s}=\frac{\sin 2 \omega(s+\alpha+1 / 4) \sin 2 \omega(s+\beta+1 / 4)}{\sin 2 \omega(s+1 / 4) \sin 2 \omega(s+3 / 4)}, \\
\lambda_{n}=-4 \sin \omega n \sin \omega(n+2 \alpha+2 \beta-1), \\
x_{s}=2 \cos 2 \omega(s+1 / 4) .
\end{gathered}
$$

Under the conditions (4.3) or (4.4), this difference equation can be transformed by a gauge transformation $P_{n}\left(x_{s}\right)=\chi(s) \psi_{n}(s)$ with an appropriate periodic function $\chi(s)$ to the hermitian form

$$
U_{s+1} \psi_{n}(s+1)+U_{s} \psi_{n}(s-1)-\left(A_{s}+C_{s}\right) \psi_{n}(s)=\lambda_{n} \psi_{n}(s),
$$

where $U_{s}=\sqrt{A_{s} C_{s-1}}$ is a real function because $A_{s} C_{s-1}>0$ for all integer $s$. Hence (4.7) provides a nontrivial example of the Hermitian Jacobi matrix with periodic coefficients whose eigenfunctions and eigenvalues are known explicitly. Note that in this case, the difference equation for symmetric polynomials is determined by the tridiagonal Jacobi matrix (cf. [17]).

The weight function for the corresponding AWP has the form

$$
w_{s}=R \sin 2 \omega(s+1 / 4) \prod_{k=1}^{s} \frac{\sin 2 \omega(k+\alpha-3 / 4) \sin 2 \omega(k+\beta-3 / 4)}{\sin 2 \omega(k-\alpha+1 / 4) \sin 2 \omega(k-\beta+1 / 4)},
$$

where $s=0,1, \ldots, N-1$, and $R$ is a normalization constant.

One can check that $w_{s}>0$ for the intervals (4.3) and (4.4) as it should be (by the Favard theorem) for the case of positive recurrence coefficients $u_{n}$. As in the generic case, the considered symmetric polynomials do not obey complete self-duality. 


\section{Finite orthogonality relation for continuous $q-J a c o b i$ poly- nomials}

In this section we study a finite set of the continuous $q$-Jacobi polynomials $[3,9,12]$ for $q$ a root of unity. These polynomials appear from the AWP with arbitrary parameters $a$ and $c$ and the choice

$$
b=a q^{1 / 2}, \quad d=c q^{1 / 2}
$$

(we use a slightly different parametrization than in $[3,9,12]$ ). Moreover, we will assume that $M$ in (1.12) is odd. Then from (2.5) we see that $E_{N}=0$, hence the zeros of the $N$ th continuous $q$-Jacobi polynomials are

$$
x_{s}=2 \cos \pi \frac{M}{N}(s+1 / 2)=q^{(s+1 / 2) / 2}+q^{-(s+1 / 2) / 2}, \quad s=0,1, \ldots, N-1 .
$$

We could set $M=1$ in (5.2). However this parametrization is more convenient for further calculations. The zeros (5.2) are simple, real, and they coincide with zeros of the monic Chebyshev polynomials of the first kind $T_{N}(x)=2 \cos N \theta$.

In order to simplify the expression for $P_{N-1}\left(x_{s}\right)$, we use another form of the continuous Jacobi polynomials due to Rahman $[3,9]$

$$
P_{n}\left(x_{s}\right)=D_{n 4} \varphi_{3}\left(\begin{array}{c}
p^{-n}, p^{-s}, p^{s+1},-a c p^{n} \\
a p^{1 / 2}, c p^{1 / 2},-p
\end{array} ; p, p\right),
$$

where $p=q^{1 / 2}=e^{\pi i M / N}$ and

$$
D_{n}=\frac{(-p ; p)_{n}\left(a p^{1 / 2} ; p\right)_{n}\left(c p^{1 / 2} ; p\right)_{n}}{p^{n / 2}\left(-a c p^{n} ; p\right)_{n}}
$$

The formula (5.3) is valid for all $n=0,1, \ldots, N-1$. However, for $n=N-1$, a remarkable simplification takes place. Since $M$ is odd we have $p^{1-N}=-p$, hence $(5.3)$ is reduced to the balanced ${ }_{3} \varphi_{2}$ function

$$
P_{N-1}\left(x_{s}\right)=D_{N-13} \varphi_{2}\left(\begin{array}{c}
p^{-s}, p^{s+1}, a c p^{-1} \\
a p^{1 / 2}, c p^{1 / 2}
\end{array} ; p, p\right) .
$$

But this sum can be calculated exactly using Jackson's extension of the Pfaff-Saalschutz formula [9] and adapting it to roots of unity. After this, we have

$$
P_{N-1}\left(x_{s}\right)=D_{N-1}\left(\frac{a c}{p}\right)^{s} \frac{\left(a^{-1} p^{3 / 2} ; p\right)_{s}\left(c^{-1} p^{3 / 2} ; p\right)_{s}}{\left(a p^{1 / 2} ; p\right)_{s}\left(c p^{1 / 2} ; p\right)_{s}}
$$

The $P_{N}^{\prime}\left(x_{s}\right)$ can be easily calculated because $P_{N}(x)=2 \cos \theta N, x=2 \cos \theta$. Namely, we have

$$
P_{N}^{\prime}\left(x_{s}\right)=\frac{N(-1)^{s+(M-1) / 2}}{\sin (\pi M(s+1 / 2) / N)} .
$$

From (1.5) we find thus the weight function of the continuous $q$-Jacobi polynomials

$$
w_{s}=R_{N} \sin (\pi M(s+1 / 2) / N) \frac{(-p / a c)^{s}\left(a p^{1 / 2} ; p\right)_{s}\left(c p^{1 / 2} ; p\right)_{s}}{\left(a^{-1} p^{3 / 2} ; p\right)_{s}\left(c^{-1} p^{3 / 2} ; p\right)_{s}}, \quad s=0,1, \ldots, N-1
$$


where the normalization constant is

$$
R_{N}=i p^{-1 / 2} \frac{\left(-c p^{1 / 2} ; p\right)_{N-1}\left(-a p^{1 / 2} ; p\right)_{N-1}}{(-a c p ; p)_{N-1}(-p ; p)_{N-1}} .
$$

In general, the parameters $a, c$ are complex and the weight function (5.8) is also complex. Nevertheless, there are important cases when the weight function becomes positive. Indeed, put $M=1$ and choose the following parametrization [3]

$$
a=p^{\alpha+1 / 2}, \quad c=-p^{\beta+1 / 2},
$$

which is quite natural for it leads to the ordinary Jacobi polynomials $P_{n}^{(\alpha, \beta)}(x)$ when $N \rightarrow \infty$. Using this parametrization and passing to the trigonometric notations, we can state the following theorem.

Theorem 3. The weight function of the finite-dimensional q-analogs of the Jacobi polynomials (5.3) for $q=\exp (2 \pi i / N)$ has the form

$$
w_{s}=R_{N} \sin (\omega(2 s+1)) \prod_{k=1}^{s} \frac{\sin \omega(k+\alpha) \cos \omega(k+\beta)}{\sin \omega(k-\alpha) \cos \omega(k-\beta)},
$$

where $\omega=\pi / 2 N, s=0,1, \ldots, N-1$, and the normalization constant

$$
R_{N}=\prod_{k=1}^{N-1} \frac{\sin \omega(k+\beta) \cos \omega(k+\alpha)}{\cos \omega k \sin \omega(k+\alpha+\beta+1)} .
$$

For

$$
-1<\alpha, \beta<1
$$

the weight function (5.11) is positive, $w_{s}>0$.

Proof of the positivity of $w_{s}$ is straightforward. In this case, the recurrence coefficients $u_{n}$ are positive too and the coefficients $b_{n}$ are real. So the condition (5.13) leads to the class of ordinary orthogonal polynomials [8] having the positive weight function on the real spectral set. The particular choice $\alpha=\beta$ corresponds to the $q$-ultraspherical polynomials considered (for the case of $q$ a root of unity) in [17]. There are also nine special cases when the weight function (5.11) is reduced to simpler expressions. These cases arise when $\alpha, \beta=-1 / 2,0,1 / 2$. For example, when $\alpha=\beta=-1 / 2$ we get finite set of Chebyshev polynomials of the first kind with the constant weight function $w_{s}=1 / N$; for $\alpha=\beta=0$ we obtain Legendre $q$-polynomials with $w_{s}=\sin \omega \sin \omega(2 s+1)$; for $\alpha=-1 / 2, \beta=1 / 2$ we have the weight function $w_{s}=N^{-1} \cot \omega / 2 \cos ^{2} \omega(s+1 / 2)$, etc.

Note that the normalization condition $w_{s}=1$ can be considered as a nontrivial summation analog of the standard beta-integral, because in the limit $q \rightarrow 1$ (i.e., $N \rightarrow \infty$ ) we get the ordinary Jacobi polynomials with continuous weight function $w(x)=(1-x)^{\alpha}(1+x)^{\beta}$. Normalization of this function leads to the ordinary beta-integral. $q$-Extension of the beta integral was found in [3] when $q$ is inside the unit disk, $|q|<1$.

Consider some limiting cases. When $c \rightarrow 0$, we get the continuous $q$-Laguerre polynomials [12] with the weight function

$$
w_{s}=i p^{-1 / 2} \frac{\left(-a p^{1 / 2} ; p\right)_{N-1}}{(-p ; p)_{N-1}} \sin (\pi M(s+1 / 2) / N) a^{-s} p^{-s^{2} / 2} \frac{\left(a p^{1 / 2} ; p\right)_{s}}{\left(a^{-1} p^{3 / 2} ; p\right)_{s}} .
$$

When $a=p^{1 / 2}$, we get an interesting trigonometric identity

$$
\sum_{s=0}^{N-1} \sin \pi \frac{M}{N}(s+1 / 2) p^{-s(s+1) / 2}=-i p^{1 / 2} .
$$


If in (5.14) we take the limit $a \rightarrow 0$ then we get the weight function for $q$-Hermite polynomials

$$
w_{s}=\frac{i(-1)^{s} p^{-s^{2}-s-1 / 2}}{(-p ; p)_{N-1}} \sin (\pi M(s+1 / 2) / N) .
$$

\section{Finite orthogonality for big $q$-Jacobi polynomials}

Big $q$-Jacobi polynomials can be considered as some limiting subcase of the AWP [9]. However, it is more convenient to work directly with these polynomials themselves. Their recurrence coefficients are

$$
u_{n}=\xi_{n} \eta_{n-1}, \quad b_{n}=1-\xi_{n}-\eta_{n}
$$

where

$$
\begin{aligned}
\xi_{n} & =-a c q^{n+1} \frac{\left(1-q^{n}\right)\left(1-b q^{n}\right)\left(1-a b c^{-1} q^{n}\right)}{\left(1-a b q^{2 n}\right)\left(1-a b q^{2 n+1}\right)} \\
\eta_{n} & =\frac{\left(1-a q^{n+1}\right)\left(1-c q^{n+1}\right)\left(1-a b q^{n+1}\right)}{\left(1-a b q^{2 n+1}\right)\left(1-a b q^{2 n+2}\right)}
\end{aligned}
$$

The explicit form of the big $q$-Jacobi polynomials is

$$
P_{n}(x)=D_{n 3} \varphi_{2}\left(\begin{array}{c}
q^{-n}, a b q^{n+1}, x \\
a q, c q
\end{array} ; q, q\right),
$$

where

$$
D_{n}=\frac{(a q ; q)_{n}(c q ; q)_{n}}{\left(a b q^{n+1} ; q\right)_{n}}
$$

For $q$ fixed by (1.12), the $N$ th polynomial has the form

$$
P_{N}(x)=x^{N}-1+\frac{\left(1-a^{N}\right)\left(1-c^{N}\right)}{1-(a b)^{N}} .
$$

In what follows, we set $c=1$. Then the zeros of the polynomial $P_{N}(x)$ coincide with the roots of unity and lie at the vertices of the regular polygon

$$
x_{s}=q^{s}, \quad s=1,2, \ldots, N .
$$

For $P_{N-1}\left(x_{s}\right)$ we have

$$
P_{N-1}\left(x_{s}\right)=D_{N-12} \varphi_{1}\left(\begin{array}{c}
q^{s-N}, a b \\
a q
\end{array} ;, q\right) .
$$

This expression can be evaluated using the root-of-unity analog of the Chu-Vandermonde formula [9] yielding

$$
P_{N-1}\left(x_{s}\right)=D_{N-1}(a b)^{N-s} \frac{\left(b^{-1} q ; q\right)_{N-s}}{(a q ; q)_{N-s}}
$$

where

$$
D_{N-1}=N \frac{\left(1-a^{N}\right)\left(1-a b q^{-1}\right)}{(1-a)\left(1-(a b)^{N}\right)} .
$$

Hence for the weight function, using (1.5), we have

$$
w_{s}=\frac{\left(1-a^{N}\right)(1-a b q)(b ; q){ }_{s} q^{s}}{a q(b-1)\left(1-a^{N} b^{N}\right)\left(a^{-1} ; q\right)_{s}}, \quad s=1,2, \ldots, N .
$$


It can be verified that this weight function is normalized, $\sum_{s=1}^{N} w_{s}=1$.

There are two interesting limiting cases: when $a \rightarrow \infty$ we get the $q$-Meixner polynomials [12] with the weight function

$$
w_{s}=b^{1-N}(1-b)^{-1}(b ; q)_{s} q^{s}
$$

When $b \rightarrow 0$ we get the big $q$-Laguerre polynomials [12] with the weight function

$$
w_{s}=(q a)^{-1}\left(a^{N}-1\right) \frac{q^{s}}{\left(a^{-1} ; q\right)_{s}} .
$$

It should be noted however that in both these cases we obtain only special (one-parameter) type of the corresponding polynomials due to the condition $c=1$.

\section{Finite orthogonality for alternative $q$-Jacobi polynomials}

So far, we were considering AWP with the base $q$. In this section we consider a special class of the AWP with the base $p=q^{1 / 2}=e^{\pi i M / N}$, where $M$ is odd. These polynomials are orthogonal on some points of the real interval and can be interpreted as alternative $q$-Jacobi polynomials.

Let us set $c=-d=1$ and keep $a$ and $b$ as arbitrary complex parameters for AWP. Then the $n$th Askey-Wilson polynomial (with the base $p$ ) is written as (cf. (1.9))

$$
P_{n}(x)=D_{n 4} \varphi_{3}\left(\begin{array}{c}
p^{-n},-a b p^{n-1}, a z, a / z \\
a b, a,-a
\end{array} ; p, p\right),
$$

where $x=z+z^{-1}$ and in the expression for normalization constant $D_{n}$ (1.10) we should substitute $p$ instead of $q$. These polynomials do not belong to the family of finite polynomials considered in Section 2, but rather they are subcases of the $q$-Racah polynomials considered in [2]. Still, as far as the authors understand, the specific property of these polynomials to have positive weight functions for some particular choices of $M$ and $N$ has not been discussed in the literature (see also [16, 17]).

Let $n=N+1$. Then taking into account that $p^{N}=-1$ we get

$$
P_{N+1}(x)=D_{N+13} \varphi_{2}\left(\begin{array}{c}
p^{-N-1}, a z, a / z \\
a,-a
\end{array} ; p, p\right),
$$

where

$$
D_{N+1}=a^{-N-1}\left(1-a^{2}\right)\left(1-a^{2 N}\right) .
$$

In order for polynomials $P_{n}(x), 0 \leq n \leq N+1$, to be well defined we impose the constraints

$$
a b, a, b \neq p^{k}, \quad k=0,1, \ldots, 2 N-1 .
$$

In particular, in this case $u_{n}$ does not vanish between two zeros at $n=0$ and $n=N+1$.

The expression (7.2) can be simplified using the Jackson summation formula [9]

$$
P_{N+1}(x)=(z ; p)_{N+1}\left(z^{-1} ; p\right)_{N+1}=z^{-N-1}\left(z^{2}-1\right)\left(z^{2 N}-1\right) .
$$

From (7.4) we find zeros $x_{s}$ of the polynomial $P_{N+1}(x)$

$$
x_{s}=p^{s}+p^{-s}=2 \cos \pi M s / N, \quad s=0,1,2, \ldots, N .
$$

It is seen from (7.5) that all the zeros $x_{s}$ are real and simple. 
From (7.4) one can derive

$$
P_{N+1}^{\prime}\left(x_{s}\right)=2 N(-1)^{s} / \chi_{s}
$$

where $\chi_{s}$ is a "characteristic function" defined as follows

$$
\chi_{s}= \begin{cases}1, & s=1,2, \ldots, N-1 \\ 1 / 2, & s=0, N\end{cases}
$$

In order to calculate $P_{N}\left(x_{s}\right)$ note that AWP are invariant under any permutation of the parameters $a, b, c, d$. Hence we can write

$$
P_{N}\left(x_{s}\right)=D_{N 3} \varphi_{2}\left(\begin{array}{c}
p^{-s}, p^{s}, a b p^{-1} \\
a, b
\end{array} ; p, p\right)
$$

where

$$
D_{N}=\frac{(-1 ; p)_{N}(a ; p)_{N}(b ; p)_{N}}{\left(a b p^{-1} ; p\right)_{N}}
$$

Again the expression (7.7) can be simplified using the Jackson summation formula:

$$
P_{N}\left(x_{s}\right)=D_{N} \frac{(p / a ; p)_{s}(p / b ; p)_{s}}{(a ; p)_{s}(b ; p)_{s}}(a b / p)^{s}, \quad s=0,1,2, \ldots, N .
$$

Combining all these results together we can state the following theorem.

Theorem 4. The weight function of the finite set of alternative q-Jacobi polynomials (7.1) with $p=\exp (\pi i M / N),(N, M)=1, M$ odd, and restrictions upon parameters (7.3), has the form

$$
w_{s}=\frac{h_{N}}{P_{N}\left(x_{s}\right) P_{N+1}^{\prime}\left(x_{s}\right)}=A_{N} \chi_{s} \frac{(a ; p)_{s}(b ; p)_{s}(-p / a b)^{s}}{(p / a ; p)_{s}(p / b ; p)_{s}},
$$

where $s=0,1, \ldots, N$ and

$$
A_{N}=\frac{(p ; p)_{N}(-a ; p)_{N}(-b ; p)_{N}}{2 N(-a b ; p)_{N}}
$$

The normalization condition $\sum_{s=0}^{N} w_{s}=1$ in this case leads to some interesting trigonometric identities. Indeed, taking the limit $a \rightarrow 0, b \rightarrow 0$ we obtain the weight function

$$
w_{s}=\chi_{s} \frac{(p ; p)_{N}}{2 N}(-1)^{s} p^{-s^{2}}
$$

The normalization condition leads to the celebrated Gauss sum [7] (see also the Appendix)

$$
\sum_{s=0}^{N-1}(-1)^{s} p^{-s^{2}}=(-p ; p)_{N-1}=\sqrt{N} p^{N(N-1) / 4}\left(\frac{N}{M}\right),
$$

where $\left(\frac{N}{M}\right)$ is the Jacobi symbol. For $M=p_{1} p_{2} \cdots p_{K}$ - the prime number decomposition of $M-$ this symbol is defined as the product

$$
\left(\frac{N}{M}\right)=\left(\frac{N}{p_{1}}\right) \cdots\left(\frac{N}{p_{K}}\right)
$$

where $\left(\frac{N}{p_{j}}\right)$ is the Legendre symbol. The latter is equal to +1 if $N$ is a quadratic residue (i.e., if there exists an integer $x$ such that $\left.x^{2}=N\left(\bmod p_{j}\right)\right)$, to -1 if $N$ is not a quadratic residue, and to 0 if $p_{j}$ divides $N$. In the simplest case $M=1$, one has $\left(\frac{N}{1}\right)=1$. 
In another limiting case, setting $a=p^{1 / 2}$ and taking the limit $b \rightarrow 0$, we get the weight function

$$
w_{s}=A_{N} \chi_{s} p^{-s^{2} / 2}
$$

where

$$
A_{N}=\frac{(p ; p)_{N}\left(-p^{1 / 2} ; p\right)_{N}}{2 N}=\frac{\left(-p^{1 / 2} ; p\right)_{N}}{(-p ; p)_{N-1}} .
$$

Using the formulas (8.16) and (8.19) from the Appendix, one can simplify the expression (7.13) for $A_{N}$ :

$$
A_{N}=\sqrt{\frac{2}{N}} p^{N / 4}\left(\frac{2 N}{M}\right) .
$$

Hence we have the following trigonometric Gauss-like sum

$$
\sum_{s=0}^{N-1} p^{-s^{2} / 2}=\frac{1}{2}\left(1-(-i)^{M N}\right)+\sqrt{\frac{N}{2}} \exp (-i \pi M / 4)\left(\frac{2 N}{M}\right) .
$$

For $M=1$ this identity was derived in [13]. The $M \neq 1$ cases were probably discussed in the literature as well.

At the final stages of preparation of this paper, the authors have discovered that in $1968 \mathrm{M}$. W. Wilson had considered a discrete analog of the Legendre polynomials [18], which is a particular subcase of the above alternative $q$-Jacobi polynomials. Indeed, let us set in $(7.9) a=-b=p^{1+\epsilon}$ and take the limit $\epsilon \rightarrow 0$. As a result, one finds the weight function $w_{0}=w_{N}=0$ and $w_{s}=$ $\tan (\pi M / 2 N) \sin (\pi M s / N)$ for $s=1,2, \ldots, N-1$. For $M=1$, it coincides with the weight function of [18] up to a normalization constant. Although some important characteristics of the corresponding polynomials (e.g., the nice approximation properties) have been found in [18] the description was not complete. In particular, the explicit form of the recurrence coefficients, which has not been derived, is

$$
u_{n}=\frac{\sin ^{2} \pi n / 2 N \cos \pi(n-1) / 2 N \cos \pi(n+1) / 2 N}{\sin \pi(2 n-1) / 2 N \sin \pi(2 n+1) / 2 N}, \quad b_{n}=0 .
$$

Only the first three coefficients $u_{1,2,3}$ were found in [18] and they coincide with (7.16). Note that the corresponding polynomials are $q$-analogs of the Legendre polynomials different from those mentioned above which were considered in [17]. The latter are simpler but it is natural to expect that they provide a similar approximation of the Legendre polynomials for $N \rightarrow \infty$.

Returning to the general weight function (7.9), we note that when $a=p^{\alpha}, b=-p^{\beta}$, and $0<$ $\alpha, \beta<1$, the recurrence coefficients $u_{n}$ become positive leading to the positivity of the corresponding weight function $w_{s}, s=0,1, \ldots, N$. Verification of this statement is elementary. In the limit $p \rightarrow 1$ we recover the ordinary Jacobi polynomials $P_{n}^{(\alpha-1, \beta-1)}(x)$. We have thus a subclass of the "classical" orthogonal polynomials (in the sense of positivity of the weight function on a real interval) among the alternative $q$-Jacobi polynomials in the same way as for the case of the continuous $q$-Jacobi polynomials.

Although we did not give a complete classification of the regions of complex parameters $a, b, c, d$ and $q$ in the recurrence coefficients (1.6) leading to positive measures for the corresponding (associated) Askey-Wilson polynomials, the present work provides some necessary tools for the resolution of this important problem.

The authors are deeply indebted to R. Askey and G. Gasper for helpful remarks to the preliminary version of this paper. 


\section{Appendix}

In this appendix we present some useful formulas concerning $q$-calculus when $q$ is a root of unity. Some of them are well known, nevertheless they are included here because the systematic treatment of such formulas is absent in the literature. We consider here only standard $q$-hypergeometric series, although there are similar relations for the bilateral series as well.

Assume that $q$ is a primitive root of unity, i.e., $q=e^{2 \pi i M / N}$, where $(N, M)=1$. Denote also $p=q^{1 / 2}=e^{\pi i M / N}$. The first set of formulas is connected to the $q$-shifted factorial defined in the standard manner

$$
(a ; q)_{n}=(1-a)(1-a q) \ldots\left(1-a q^{n-1}\right), \quad(a ; q)_{0}=1 .
$$

Obviously $(a ; q)_{n+N}=(a ; q)_{n}(a ; q)_{N}$, so it is sufficient to study only the case $0 \leq n \leq N$. The following identities are easy to derive

$$
(a ; q)_{N}=1-a^{N}
$$

(in particular $(a ; q)_{N}=0$ if $a$ is a primitive $N$ th root of unity) and

$$
\begin{gathered}
(a ; q)_{N-1}=\frac{1-a^{N}}{1-a q^{-1}}, \quad \text { if } a \neq q, \\
(q ; q)_{N-1}=N .
\end{gathered}
$$

In general, there are no simple formulas for $(a ; p)_{N}$ and $(a ; p)_{N-1}$ like (8.2) and (8.3). In our case the following relations for inversion of the parameter are valid. Assume that $M$ is odd. Then

$$
\begin{array}{r}
(a ; p)_{N}=a^{N} p^{-N(N+1) / 2}\left(-a^{-1} p ; p\right)_{N} \\
(a ; p)_{N-1}=a^{N-1} p^{1-N(N+1) / 2}\left(-a^{-1} p^{2} ; p\right)_{N-1} .
\end{array}
$$

If $M$ is even (hence $N$ is odd), then

$$
\begin{array}{r}
(a ; p)_{N}=-a^{N}\left(a^{-1} p ; p\right)_{N}, \\
(a ; p)_{N-1}=p a^{N-1}\left(a^{-1} p^{2} ; p\right)_{N-1} .
\end{array}
$$

The next set of formulas is connected to finite sums involving the $q$-shifted factorial. The simplest one is a special case of the $q$-binomial theorem which can be written as

$$
{ }_{1} \varphi_{0}\left(q^{s} ; q, z\right)=\sum_{k=0}^{N-s} \frac{\left(q^{s} ; q\right)_{k}}{(q ; q)_{k}} z^{k}=\frac{1-z^{N}}{(z ; q)_{s}}=\left(z q^{-1} ; q^{-1}\right)_{N-s},
$$

where $s=1,2, \ldots, N-1$. In (8.9) and below we use the standard notation for $q$-hypergeometric functions although we always assume that only the first $N$ terms are present in the corresponding series.

The Chu-Vandermonde formula [9] for $q$ a root of unity looks like this:

$$
{ }_{2} \varphi_{1}\left(\begin{array}{c}
q^{s}, a \\
c
\end{array} ; q, q\right)=\frac{a^{N}-c^{N}}{1-c^{N}} \frac{(q / c ; q)_{s}}{(q a / c ; q)_{s}}, \quad s=1,2, \ldots, N-1,
$$

where $a, c$ are arbirary complex parameters, $c^{N} \neq 1$. In particular, for $s=1$ we get a pretty summation formula

$$
\sum_{k=0}^{N-1} \frac{(a ; q)_{k}}{(c ; q)_{k}} q^{k}=\frac{\left(a^{N}-c^{N}\right)\left(1-c q^{-1}\right)}{\left(1-c^{N}\right)\left(a-c q^{-1}\right)} .
$$


Similarly, Jackson's extension of the Pfaff-Saalschutz sum [9] in this special case is written as

$$
\sum_{k=0}^{N-1} \frac{(a ; q)_{k}(b ; q)_{k} q^{k}}{(c ; q)_{k}\left(a b c^{-1} q^{2} ; q\right)_{k}}=\frac{\left(a^{N}-c^{N}\right)\left(b^{N}-c^{N}\right)(q-c)(a b q-c)}{\left(1-c^{N}\right)\left(a^{N} b^{N}-c^{N}\right)(q a-c)(q b-c)} .
$$

The Dixon $q$-summation formula [9] takes the form

$$
\sum_{k=0}^{N-1} \frac{(-q a ; q)_{k}(b ; q)_{k}}{(-a ; q)_{k}\left(a^{2} b^{-1} q ; q\right)_{k}}(a / b)^{k}=\frac{\left(b-a^{2}\right)\left(1+a^{N}\right)\left(b^{N}-a^{N}\right)}{(b-a)(1+a)\left(b^{N}-a^{2 N}\right)} .
$$

A special case of the Singh quadratic transformation yields, for $q^{N}=1$, the summation formula

$$
{ }_{4} \varphi_{3}\left(\begin{array}{c}
q, a, a / p, a^{2} q / b^{2} \\
a^{2},-a b^{-1} q,-a b^{-1} p^{3}
\end{array} ; q, q\right)=\frac{(-p ; p)_{N-1}(b / p ; p)_{N-1}}{(-a ; p)_{N-1}(b / a ; p)_{N-1}},
$$

where we assume that $M$ is odd so that $p^{N}=-1$. If one takes the limit $a \rightarrow 0, b \rightarrow 0, b / a \rightarrow 0$ then we get an important summation formula

$$
\sum_{k=0}^{N-1}(-1)^{k} q^{-k^{2} / 2}=(-p ; p)_{N-1},
$$

going back to the works of Gauss in the beginning of nineteenth century. The Gauss sum (8.15) plays a prominent role in some problems of the number theory [7]. The right hand side of (8.15) can be rewritten as follows $[7]$

$$
(-p ; p)_{N-1}=\left(\frac{N}{M}\right) \sqrt{N} e^{\pi i M(N-1) / 4},
$$

where $\left(\frac{N}{M}\right)$ is the Jacobi symbol. Analogously, considering the Gauss sum

$$
\sum_{k=0}^{2 N-1}(-1)^{k} p^{-k^{2} / 2}=\left(-p^{1 / 2} ; p^{1 / 2}\right)_{2 N-1},
$$

one can get the identity

$$
\left(-p^{1 / 2} ; p^{1 / 2}\right)_{2 N-1}=\left(\frac{2 N}{M}\right) \sqrt{2 N} e^{\pi i M(2 N-1) / 4} .
$$

From this relation and the obvious equality

$$
(-p ; p)_{N-1}\left(-p^{1 / 2} ; p\right)_{N}=\left(-p^{1 / 2} ; p^{1 / 2}\right)_{2 N-1}
$$

follows another interesting identity

$$
\left(-p^{1 / 2} ; p\right)_{N}=\frac{\left(-p^{1 / 2} ; p^{1 / 2}\right)_{2 N-1}}{(-p ; p)_{N-1}}=\left(\frac{2}{M}\right) \sqrt{2} e^{\pi i M N / 4}=(-1)^{\left(M^{2}-1\right) / 8} \sqrt{2} e^{i \pi M N / 4},
$$

where the following properties of the Jacobi symbol were used

$$
\left(\frac{m}{M}\right)\left(\frac{n}{M}\right)=\left(\frac{m n}{M}\right), \quad\left(\frac{2}{M}\right)=(-1)^{\left(M^{2}-1\right) / 8} .
$$




\section{References}

[1] W. Al-Salam, W. R. Allaway, and R. Askey, Sieved ultraspherical polynomials, Trans. Amer. Math. Soc. 284 (1984), 39-55.

[2] R. Askey and J. Wilson, A set of orthogonal polynomials that generalize the Racah coefficients or 6 - j symbols, SIAM J. Math. Anal. 10 (1979), 1008-1016.

[3] R. Askey and J. Wilson, Some basic hypergeometric orthogonal polynomials that generalize Jacobi polynomials, Mem. Amer. Math. Soc. 54 (1985), 1-55.

[4] F. V. Atkinson, "Discrete and continuous boundary problems" in Mathematics in Science and Engineering, 8, Academic Press, New York, 1964.

[5] E. Bannai and T. Ito, Algebraic Combinatorics I. Association Schemes, Benjamin/Cummings, Menlo Park, 1984.

[6] R. D. Carmichael, The general theory of linear q-difference equations, Amer. J. Math. 34 (1912), 147-168.

[7] K. Chandrasekharan, Introduction to Analytic Number Theory, Grundlehren Math. Wiss. 148, Springer-Verlag, New York, 1968.

[8] T. S. Chihara, An Introduction to Orthogonal Polynomials, Math. Appl. 13, Gordon and Breach, New York, 1978.

[9] G. Gasper and M. Rahman, Basic Hypergeometric Series, Encyclopedia Math. Appl. 35, Cambridge University Press, Cambridge, 1990.

[10] D. Gupta and D. Masson, Contiguous relations, continued fractions and orthogonality, to appear in Trans. Amer. Math. Soc.

[11] M. E. H. Ismail and M. Rahman, The associated Askey-Wilson polynomials, Trans. Amer. Math. Soc. 328 (1991), 201-237.

[12] R. Koekoek and R. F. Swarttouw, The Askey scheme of hypergeometric orthogonal polynomials and its q-analog, Delft University Technical Report 94-05, 1994.

[13] N. M. Korobov, Exponential Sums and their Applications, Math. Appl. (Soviet Ser.) 80, Kluwer, Dordrecht, 1992.

[14] D. A. Leonard, Orthogonal polynomials, duality, and association schemes, SIAM J. Math. Anal. 13 (1982), 656-663.

[15] A. F. Nikiforov, S. K. Suslov and V. B. Uvarov, Classical Orthogonal Polynomials of Discrete Variable, Springer Ser. Comput. Phys., Springer-Verlag, Berlin, 1991.

[16] V. Spiridonov and A. Zhedanov, Discrete Darboux transformations, the discrete time Toda lattice, and the Askey-Wilson polynomials, Methods Appl. Anal. 2 (1995), 369-398.

[17] V. Spiridonov and A. Zhedanov, q-Ultraspherical polynomials for $q$ a root of unity, Lett. Math. Phys. 37 (1996), 173-180. 
[18] M. W. Wilson, On a new discrete analogue of the Legendre polynomials, SIAM J. Math. Anal. 3 (1972), 157-169.

V. Spiridonov: Centre de Recherches Mathématiques, Université de Montréal, C.P. 6128, succ. Centre-ville, Montréal (Québec) H3C 3J7, Canada; current address: Laboratory of Theoretical Physics, JINR, Dubna 141980 Moscow region, Russia

A. Zhedanov: Donetsk Institute for Physics and Technology, Donetsk 340114, Ukraine 